\title{
Farmacovigilancia de psicofármacos en una farmacia de A Coruña (España)
}

\author{
Patricia Alonso Lovera \\ Farmacéutica comunitaria en A Coruña.
}

\section{PALABRAS CLAVE}

Reacción adversa a medicamentos, farmacovigilancia, farmacia comunitaria, antidepresivos, antipsicóticos, conocimiento

\section{ABREVIATURAS}

AEMPS: Agencia Española de Medicamentos y Productos Sanitarios AF: atención farmacéutica ATC: clasificación

Anatómica, Terapéutica y Química

CAFV: Centro Autonómico de Farmacovigilancia CFVG: Centro de Farmacovigilancia Gallega CGCOF: Consejo General de Colegios Oficiales de Farmacéuticos

DE: desviación estándar EA: Efectos adversos ISRS: inhibidores selectivos de la recaptación serotonina FC: farmacia comunitaria FV: farmacovigilancia HTA: hipertensión arterial IPM: información personalizada sobre el medicamento

OMS: Organización Mundial de la Salud

PA: presión arterial

PRM: problemas

relacionados con los medicamentos

RAM: reacciones adversas a medicamentos

\section{KEYWORDS}

Adverse drug reaction, pharmacovigilance, community pharmacy, antidepressants, antipsychotics, knowledge

\section{RESUMEN}

Objetivos: Realizar una actividad de farmacovigilancia (FV) en una farmacia comunitaria, registrando las reacciones adversas (RAM) y derivando a los pacientes a su médico si fuese necesario.

Material y métodos: Estudio observacional transversal. En un cuestionario ad hoc se recogieron datos demográficos de los pacientes sobre la utilización de los medicamentos, percepción sobre efectividad y seguridad de los fármacos a estudiar. Se seleccionaron cuatro principios activos: duloxetina, venlafaxina, quetiapina y olanzapina por ser de dispensación frecuente en la farmacia y de reciente comercialización.

Resultados: 47 pacientes utilizaban uno de los antidepresivos estudiados. 34 (72,3\%) mujeres y $13(27,7 \%)$ hombres. $21(55,3 \%)$ tomaban venlafaxina y $26(44,7 \%)$ duloxetina. Media de edad 52,9 (DE= 13,7) años (21-80). 36 en tratamiento con los dos antipsicóticos del estudio. $21(58,3 \%)$ utilizaban olanzapina y $15(41,8 \%)$ quetiapina. Edad media 41,6 $(\mathrm{DE}=13,4)$ años (27-70).

174 RAM en los antidepresivos y 120 en los antipsicóticos. Sequedad de boca, temblores, hipersudoración, flatulencia y ansiedad son las más frecuentes en los dos antidepresivos y sequedad de boca, reducción de la libido y aumento de peso en los dos antipsicóticos.

Se notificó 1 caso al Centro Gallego de Farmacovigilancia. Se derivaron dos pacientes al médico por problemas relacionados con la medicación (PRM).

Conclusiones: Mediante una actividad de FV en una farmacia comunitaria de A Coruña se ha detectado un elevado número de RAM, contribuyendo a un resultado más seguro de la farmacoterapia de los pacientes.

\section{Pharmacovigilance of psychotropic drugs in a pharmacy in A Coruna (Spain)}

\section{ABSTRACT}

Objectives: To carry out pharmacovigilance $(\mathrm{PhV})$ in a community pharmacy, recording the adverse reactions (ADR) and to refer patients to their doctor if necessary.

Methodology: Transversal observational study. In an ad hoc questionnaire, patients' demographic information is collected, along with details about their use of medication, and perceptions about the effectiveness and safety of drugs being studied. Four drugs were selected: duloxetine, venlafaxine, quetiapine and olanzapine, all of which were frequently dispensed in the pharmacy and recently came onto the market.

Results: 47 patients used one of the antidepressants studied. 34 (72.3\%) women and 13 (27.7\%) men. 21 (55.3\%) were taking venlafaxine and 26 (44.7\%) duloxetine. Mean age 52.9 $(\mathrm{SD}=13.7)$ years $(21-80) .36$ were undergoing treatment using two of the antipsychotics in the study. $21(58.3 \%)$ used olanzapine and $15(41.8 \%)$ quetiapine. mean age $41.6(\mathrm{SD}=13.4)$ years (27-70).

174 ADR in the antidepressants and 120 in the antipsychotics. Dry mouth, tremors, excessive sweating, flatulence and anxiety were the most common ADR in the two antidepressants and dry mouth, decreased libido and weight gain were the most common in the two antipsychotics.

One case was reported to the Galician Pharmacovigilance Center. Two patients were referred to their doctors for drug related problems (DRPs).

Conclusions: PhV activity in a community pharmacy in A Coruña detected a large number of ADR, contributing to safer pharmacotherapy outcomes for patients.

Este trabajo forma parte de la investigación realizada como trabajo final del Máster de Atención Farmacéutica Integral realizado en la Universidad de Barcelona (2013/2015).

Financiación: Ninguna ajena.

Conflicto de intereses: La autora declara no existir conflicto de intereses en relación con el contenido del artículo. Cite este artículo como: Alonso P. Farmacovigilancia de psicofármacos en una farmacia de A Coruña (España). Farmacéuticos Comunitarios. 2016 Mar 01; 8(1):5-12. doi:10.5672/FC.2173-9218.(2016/Nol8).001.02 Correspondencia: Patricia Alonso Lovera (palonso@cofc.es).

ISSN 1885-8619 @ SEFAC (Sociedad Española de Farmacia Familiar y Comunitaria). Todos los derechos reservados. 


\section{Introducción}

En el Real Decreto 577/2013 (1) se define reacción adversa a los medicamentos (RAM) como cualquier respuesta nociva y no intencionada a un medicamento, incluyendo así las reacciones adversas derivadas de cualquier uso al margen de los términos de la autorización de comercialización, abuso y errores de medicación. En el RD se potencia la vigilancia de los medicamentos de reciente comercialización y de aquellos en los que se identifique un potencial problema de seguridad que conlleve la necesidad de realizar estudios o medidas específicas para minimizar el riesgo. Estos medicamentos bajo seguimiento adicional tendrán un distintivo en la ficha técnica y prospecto para que tanto el profesional sanitario como el ciudadano prioricen la notificación de sospechas de reacciones adversas.

La farmacovigilancia (FV) es la actividad de salud pública destinada a analizar y gestionar los riesgos de los medicamentos una vez comercializados. Está orientada a la identificación, cuantificación, evaluación de los riesgos asociados al uso de los medicamentos.

Los farmacéuticos, como profesionales de la salud, están llamados a colaborar en las actividades de FV, como se ha recogido en sucesivas normas: desde la Orden de 12 de noviembre de 1973 (2), del Ministerio de Gobernación, por la que se establecía la FV por primera vez en España, hasta la Ley 44/2003, de 21 de noviembre, de Ordenación de las Profesiones Sanitarias (3). En el artículo 6.2 b de esta ley se cita entre las funciones del farmacéutico la de colaborar en los procesos farmacoterapéuticos y de vigilancia de salud pública.

La depresión es una enfermedad mental cada vez más común. Según la Organización de la Salud (OMS), el $75 \%$ de las personas que la padecen vive en los países en desarrollo (4). $\mathrm{Su}$ prevalencia se estima entre el 3\% y el 5\% y a lo largo de la vida puede afectar a entre un 10 y un 20\% de la población adulta, con un riesgo 2-3 veces mayor en mujeres que en varones. El aumento de pacientes con depresión es continuo desde hace años y no parece fácil delimitar con rigor si realmente van aumentando estos trastornos o se van diagnostican cada vez más. En general, se estima que cerca del 90\% de los pacientes depresivos jamás son visitados por un médico y por consiguiente no pueden ser diagnosticados y tratados (4).

En la farmacoterapia de la depresión se incluyen varios grupos terapéuticos (5), entre ellos los inhibidores de la recaptación de serotonina y noradrenalina: duloxetina y venlafaxina. Las principales RAM de ambos fármacos son: Síndrome serotoninérgico (cambios del estado mental: agitación, alucinaciones, coma; inestabilidad autonómica: taquicardia, tensión arterial lábil, hipertermia; aberraciones neuromusculares: hiperreflexia, descoordinación y síntomas gastrointestinales: náuseas, vómitos, diarrea); glaucoma de ángulo cerrado; elevación de la tensión arterial y de la frecuencia cardiaca, particularmente con dosis elevadas; convulsiones; hiponatremia y/o síndrome de secreción inadecuada de hormona antidiurética; sangrado anormal por reducción de la función plaquetaria; elevación del colesterol sérico; acatisia/agitación psicomotriz; sequedad bucal; alteraciones en la capacidad para conducir y utilizar máquinas (6-9).

La esquizofrenia es la psicosis primaria más común. Se trata de un trastorno grave que empieza en la adolescencia tardía o en la primera juventud y afecta más o menos por igual a ambos sexos. Se caracteriza en general por distorsiones fundamentales y típicas del pensamiento y de la percepción, junto con una afectividad inadecuada. El delirio (creencia fuerte en ideas que son falsas y sin ningún fundamento en la realidad) y las alucinaciones (especialmente las auditivas, por ejemplo, oír voces) son características psicóticas típicas de este trastorno. Puede seguir un curso crónico o recurrente, con síntomas residuales y recuperación social incompleta. Dosis elevadas de antipsicóticos aumentan el riesgo de reacciones adversas sin proporcionar más ventajas (5-8).

Olanzapina y quetiapina forman parte de los denominados "antipsicóticos atípicos”. Un elemento común a todos ellos es su menor propensión a provocar extrapiramidalismo, lo que ofrece la posibilidad de administrar dosis más altas con menos riesgo, aunque no sucede lo mismo con otros efectos neurológicos o metabólicos (10).
Los efectos secundarios de los antipsicóticos incluyen reacciones neurológicas, anticolinérgicas, metabólicas, cardiovasculares, hematológicas, etc. Entre las reacciones neurológicas se incluyen efectos de tipo parkinsoniano: temblor de reposo, acinesia, rigidez, distonías agudas (espasmos musculares prolongados, lentos); acatisia (sentimiento subjetivo de agitación); síndrome neuroléptico maligno (fiebre, sudoración, confusión, aumento de la presión arterial y del pulso, rigidez muscular, elevación de la creatina-cinasa, insuficiencia renal); discinesia tardía (movimientos involuntarios anormales de la lengua, la cabeza, la cara, la boca); y convulsiones. Entre las anticolinérgicas encontramos efectos periféricos: sequedad de boca, visión borrosa, estreñimiento, retención urinaria y efectos centrales (agitación grave y confusión) (11-13).

La incorporación de las actividades de FV a la labor diaria del farmacéutico comunitario (FC) es de gran trascendencia, pues es el profesional sanitario más próximo al paciente, con una visión global de su farmacoterapia por lo que puede identificar posibles RAM de tratamientos prescritos por diferentes especialistas y también de medicamentos sin prescripción médica.

\section{Objetivos}

Objetivo general

- Realizar una actividad de farmacovigilancia en una farmacia comunitaria, registrando las RAM observadas y derivando a los pacientes a su médico con el fin de mejorar la seguridad del uso de los medicamentos.

\section{Objetivos específicos}

- Cuantificar las sospechas de RAM referidas por los pacientes de los medicamentos elegidos.

- Derivar al médico en los casos que se considere importante para su salud.

- Informar a los pacientes de los posibles efectos adversos de dichos fármacos para prevenir la aparición de problemas relacionados con su medicación, resolviendo sus dudas acerca de ésta.

- Cuantificar el número de sospechas de RAM notificadas al Centro Autonómico de FV. 


\section{Material y métodos \\ Diseño}

Estudio observacional, analítico, prospectivo, no aleatorizado. Se realizó una intervención derivando al médico y se siguió el resultado de dicha intervención.

\section{Ámbito y duración}

El estudio se realizó en una farmacia comunitaria de la provincia de A Coruña. Los pacientes se incorporaron al estudio desde el día 15 de agosto de 2014 hasta el día 15 de marzo de 2015.

\section{Medicamentos objeto de estudio}

Se seleccionaron cuatro principios activos de presencia significativa en la farmacia comunitaria y comercializados en los últimos diez años, que se indican con denominación común internacional (mes y año de comercialización), código de clasificación anatomoterapéutica y denominación del grupo (6):

- Venlafaxina (junio 2005). N06AX16. Otros antidepresivos.

- Duloxetina (junio 2009). N06AX2. Otros antidepresivos.

- Olanzapina (agosto 2009). N05AH03. Antipsicóticos: diazepinas, oxazepinas y tiazepinas.

- Quetiapina (octubre 2008). N05AH04. Antipsicóticos: diazepinas, oxazepinas y tiazepinas.

\section{Sujetos}

Criterios de inclusión: se incluyeron en el estudio todos los pacientes de la farmacia que estaban en tratamiento con duloxetina, venlafaxina, olanzapina o quetiapina desde más de dos meses antes del inicio del estudio y acudian a retirar personalmente el medicamento

Criterios de exclusión: pacientes que iniciaban tratamiento con los fármacos de estudio. Los que no recogían personalmente el medicamento (cuidadores). Aquellos que no estaban capacitados para responder al cuestionario.

Con el fin de proteger su intimidad personal se realizó una codificación de las hojas de recogida de datos asignando un código identificativo a cada uno de los pacientes reclutados. Los pacientes podian abandonar el estudio en cualquier momento.

\section{Procedimiento}

Al comienzo del estudio se revisaron las fichas farmacoterapéuticas de los pacientes habituales en la farmacia y se contactó con ellos para administrarles el cuestionario. Se propuso también a todos los pacientes no habituales de la farmacia que cumplían las condiciones de inclusión.

Las entrevistas se realizaron en la zona de atención personalizada de la farmacia. Al mismo tiempo se resolvían las dudas de los pacientes acerca de su medicación y se les informaba de todos los posibles adversos que pudiesen aparecer a lo largo del tratamiento.

No se realizó el cálculo de tamaño muestral, ya que se ofreció participar a toda la población diana accesible; es decir, a todos los pacientes de la farmacia que estaban siendo medicados con los fármacos a estudiar.

\section{Variables}

- Características demográficas de los pacientes: edad, sexo, IMC, medicamentos utilizados de manera continuada o esporádica, alergias y otras características del uso de los medicamentos.

- Número de RAM detectadas.

- Número de pacientes a los que se les detectaron RAM.

- Número de notificaciones de sospechas de RAM.

- Número de sospechas de RAM detectadas para cada uno de los medicamentos en estudio.

\section{Instrumentos de registro}

y medida

- Hoja de registro de datos (figuras 1 y 2).

- Tarjetas amarillas de notificación espontánea.

- Báscula electrónica calibrada con medida de peso y altura.

Se utilizaron como herramientas de consulta de las RAM la mayor parte de las fuentes primarias, secundarias y terciarias reflejadas en el apartado de referencias bibliográficas.

\section{Resultados}

Se entrevistó a 83 pacientes, de los cuales 47 utilizaban uno de los antidepresivos del estudio. De ellos 34 eran mujeres y 13 hombres. 21 $(55,3 \%)$ tomaban venlafaxina, 14 $(66,7 \%)$ mujeres y $7(33,3 \%)$ hombres y $26(44,7 \%)$ duloxetina, 20 (76,9\%) mujeres y $6(23,1 \%)$ hombres. La media de edad fue de 52,9 $(\mathrm{DE}=13,7)$ años (21-80).

Los otros 36 estaban en tratamiento con uno de los dos antipsicóticos del estudio. 21 (58,3\%) utilizaban olanzapina, $12(57,1 \%)$ mujeres y $9(42,8 \%)$ hombres y $15(41,8 \%)$ quetiapina, $11(73,3 \%)$ mujeres y 4 $(26,7 \%)$ hombres. La edad media fue de 41,6 (DE=13,4) años (27-70).

La efectividad percibida de los antidepresivos por los pacientes fue positiva en $41(87,2 \%)$ pacientes y $6(12,7 \%)$ no notaron mejoría alguna. De los 26 pacientes que tomaron duloxetina han notado mejoría con el tratamiento $21(80,8 \%)$ y 5 $(19,2 \%)$ no han notado ninguna progresión a pesar de la medicación. De los 21 pacientes que tomaron venlafaxina han notado mejoría $20(95,2 \%)$ y sólo $1(4,8 \%)$ no ha notado mejoría.

De los antipsicóticos, en el caso de olanzapina 17 (80,9\%) pacientes notaron mejoría con el tratamiento, pero $4(19,1 \%)$ no mejoraron a pesar del tratamiento. De los 15 pacientes tratados con quetiapina, 13 (86,7\%) han mejorado pero $2(13,3 \%)$ no han experimentado mejoría.

\section{Reacciones adversas}

Se detectaron un total de 174 sospechas de RAM en 47 pacientes, media de 3,7 RAM por paciente (tablas 1 y 2). En 26 pacientes que tomaron duloxetina se detectaron 133 sospechas de RAM, media 6,7 por paciente (DE=5,2; 0-21). 41 sospechas de RAM se detectaron en 21 pacientes que tomaron venlafaxina, media de 2,0 por paciente ( $\mathrm{DE}=2,2 ; 0-9)$.

Respecto a los antipsicóticos, se detectaron un total de 120 sospechas de RAM en 36 pacientes, media 3,3 RAM por paciente. 21 pacientes que tomaban olanzapina sufrieron 78 sospechas de RAM, media 3,7 RAM (DE=3.6; 1-14) por paciente. Corresponden a quetiapina 42 sospechas de RAM en 15 pacientes, media 2,8 RAM por paciente $(\mathrm{DE}=2,0 ; 1-20)$. 


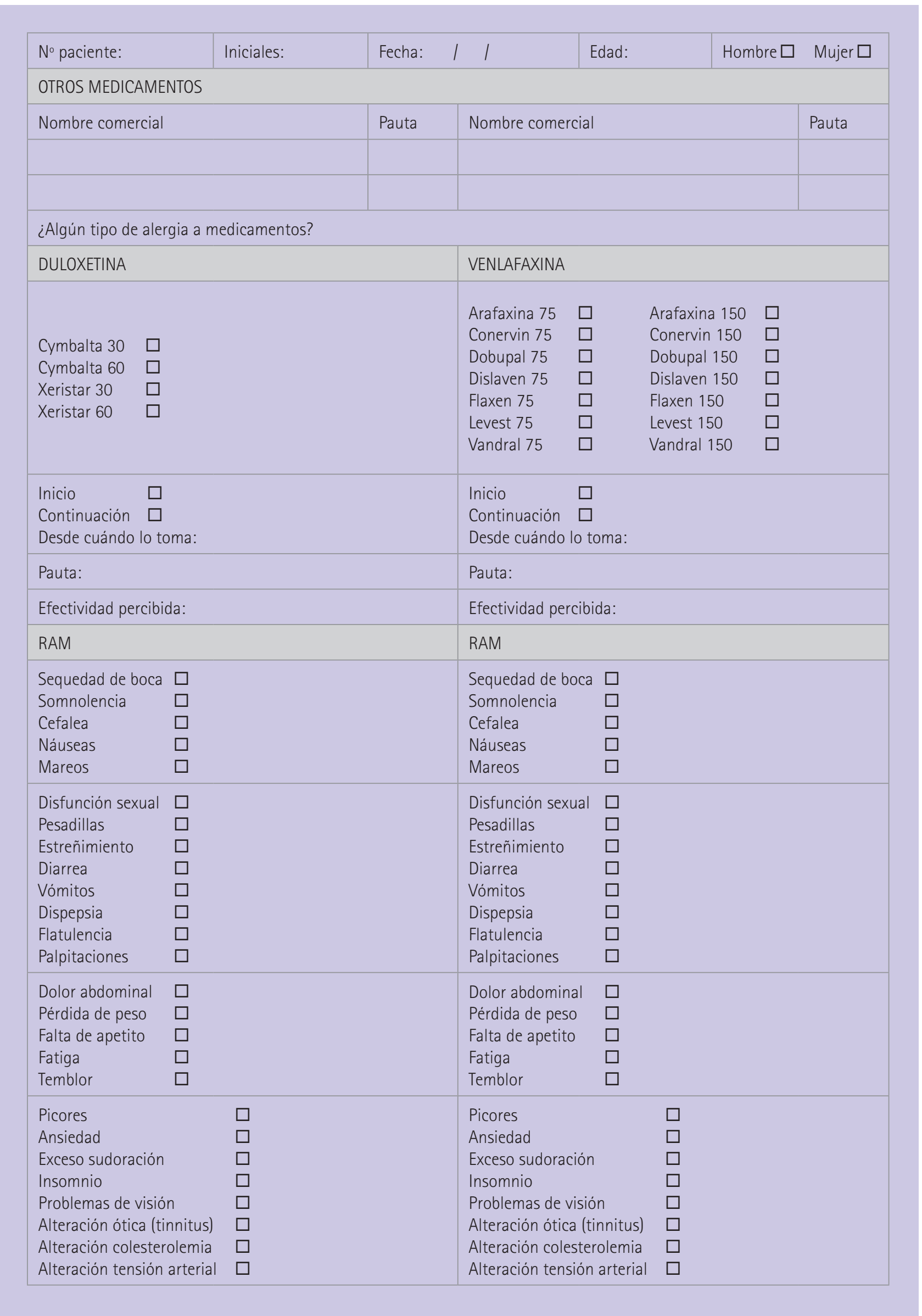

Figura 1 Hoja de registro de antidepresivos 


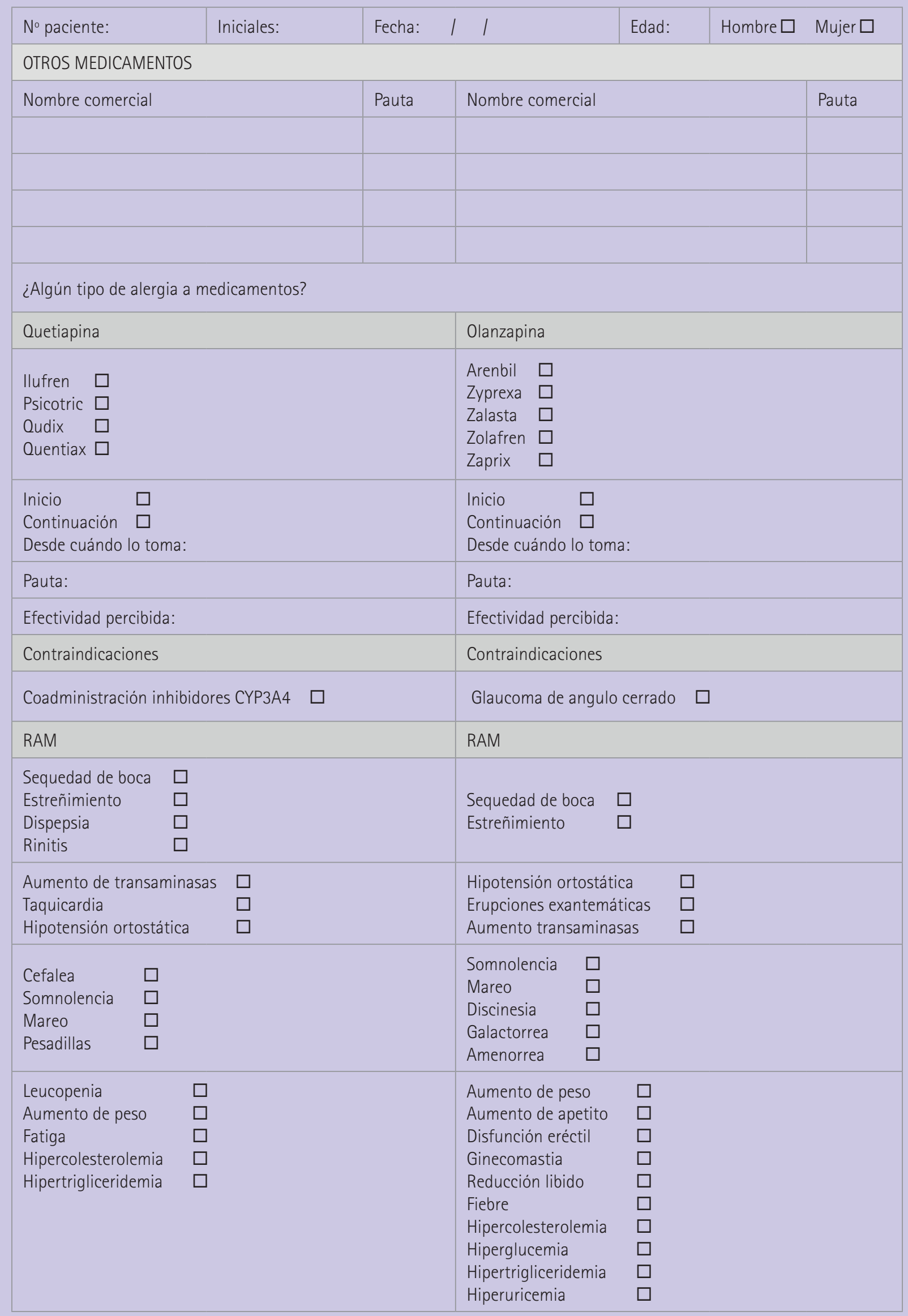


Tabla 1 Número y porcentaje de pacientes que refiere cada RAM de antidepresivos

\begin{tabular}{|c|c|c|c|}
\hline & Venlafaxina & Duloxetina & Total \\
\hline RAM & n (\%) & n (\%) & n (\%) \\
\hline Hipersudoración & $9(42,9)$ & 5 (19.2) & $14(29.8)$ \\
\hline Sequedad de boca & $6(28,6)$ & $21(80,8)$ & $27(57,4)$ \\
\hline Flatulencia & $4(19,0)$ & $10(38,5)$ & $14(29,8)$ \\
\hline Somnolencia & $3(14,3)$ & $5(19,2)$ & $8(17,0)$ \\
\hline Estreñimiento & $3(14,3)$ & $5(19,2)$ & $8(17,0)$ \\
\hline Ansiedad & $3(14,3)$ & $11(42,3)$ & $14(29,8)$ \\
\hline Cefalea & $2(9,5)$ & $5(19,2)$ & $7(14,9)$ \\
\hline Mareos & $2(9,5)$ & $6(23,1)$ & $8(17,0)$ \\
\hline Insomnio & $2(9,5)$ & $5(19,2)$ & $7(14,9)$ \\
\hline $\begin{array}{l}\text { Alteración de la tensión } \\
\text { arterial }\end{array}$ & $2(9,5)$ & $11(42,3)$ & $13(27,7)$ \\
\hline Náuseas & $1(4,8)$ & $1(3,8)$ & $2(4,3)$ \\
\hline Diarrea & $1(4,8)$ & & $1(2,1)$ \\
\hline Dispepsia & $1(4,8)$ & $5(19,2)$ & $6(12,8)$ \\
\hline Problemas de visión & $1(4,8)$ & & $1(2,1)$ \\
\hline Fatiga & $1(4,8)$ & $10(38,5)$ & $11(23,4)$ \\
\hline Palpitaciones & & $5(19,2)$ & $5(10,6)$ \\
\hline Dolor abdominal & & $5(19,2)$ & $5(10,6)$ \\
\hline Pérdida de peso & & $2(7,7)$ & $2(4,3)$ \\
\hline Pérdida de apetito & & $5(19,2)$ & $5(10,6)$ \\
\hline Temblores & & $16(61,5)$ & $16(34,0)$ \\
\hline Total RAM & 41 & 133 & 174 \\
\hline
\end{tabular}

Se notificó una sospecha de RAM al Centro Gallego de Farmacovigilancia, sin que hasta el momento se haya recibido respuesta. Se trataba de un paciente de 55 años en tratamiento con: lorazepam 1mg, (0-0-1), atorvastatina $20 \mathrm{mg}(0-0-1)$ y venlafaxina retard $150 \mathrm{mg}$ (1-0-0), prescrita hace 2 años. Desde entonces padecía un problema de hipersudoración que condicionaba su calidad de vida.

\section{Intervención}

Se informó a los pacientes sobre los posibles efectos adversos de la medicación. 2 pacientes fueron derivados al médico. En un caso (el descrito en el párrafo anterior), de hipersudoración por venlafaxina, el médico redujo la dosis (de $150 \mathrm{mg}$ a $75 \mathrm{mg}$ ), con resultado de notable mejoría en la RAM percibida. En otro caso, de hipersudoración por duloxetina, el médico no modificó el tratamiento.

En los casos de sequedad bucal se indicaron a los pacientes medidas de higiene bucal específicas, con xilitol, colutorios, pastas dentífricas, chicles, caramelos para mitigar la sensación desagradable y la recomendación de llevar una limpieza lo más exhaustiva posible para evitar caries. La mayoría de ellos han percibido notable mejoría y alivio de esta molestia.

\section{Discusión \\ Antidepresivos}

Exceso de sudoración (42,9\%) y sequedad de boca $(28,6 \%)$ son las sospechas de RAM más frecuentes en venlafaxina, seguidas de disfunción sexual y flatulencia. Se han detectado más casos de hiperhidrosis asociada al uso de otros antidepresivos, como es el caso de fluoxetina (14). En el caso de duloxetina las sospechas de RAM más frecuentes son sequedad de boca $(80,8 \%)$ y temblor $(61,5 \%)$, seguidas de ansiedad y alteración en la tensión arterial. El temblor no aparece en venlafaxina en nuestro estudio; sin embargo, se han comunicado síntomas extrapiramidales (parkinsonismo, acatisia, discinesia tardía, entre otros) inducidos por venlafaxina $\mathrm{y}$ dependiente de dosis (15).

Con respecto a la alteración de la tensión arterial y taquicardias, en el estudio no parece que tengan relevancia en venlafaxina al igual que en otras referencias bibliográficas, ya que este efecto secundario se produciría a dosis elevadas o en caso de no poder metabolizar o eliminar el fármaco (en insuficiencias hepáticas o renales) (16). Otras parecen aclarar que no hay diferencias significativas entre un antidepresivo y otro (17), ya que la depresión en sí causa alteración en la tensión arterial y no la administración del fármaco. Otras referencias indican que es duloxetina la que implica menos sospechas de alteración arterial, siendo venlafaxina la más perjudicial, a diferencia de nuestros datos (16-18).

La sequedad de boca en el caso de venlafaxina se ha detectado más en nuestro estudio $(28,6 \%)$ que en la ficha técnica, que recoge un 10\% (7), lo que puede ser debido a los otros tratamientos administrados al paciente tipo ansiolíticos que potencian la aparición de esta RAM (13).

Es importante destacar que los pacientes que tienen prescritos antidepresivos también están tomando otro tipo de medicación con similares efectos secundarios, lo que agrava la incidencia de estos; es el caso de las benzodiazepinas; las cuales provocan de por sí sequedad de boca, y muchos otros fármacos que actúan en el sistema nervioso central, así como otros antidepresivos, por ejemplo clorazepato dipotásico, clomipramina, bupropión, aripiprazol, mirtazapina, diazepam, trazodona. Se ha detectado que a mayor medicación conjunta de este tipo, mayor número de RAM detectadas que si sólo tienen prescrito el antidepresivo. El 92\% de los pacientes tratados que detectan alguna RAM toman más de un fármaco que actúa a nivel del sistema nervioso central. 
Tabla 2 Número y porcentaje de pacientes que refiere cada RAM de antipsicóticos

\begin{tabular}{|c|c|c|c|}
\hline & Olanzapina & Quetiapina & Total \\
\hline RAM & n (\%) & n (\%) & $n(\%)$ \\
\hline Sequedad de boca & $14(66.7)$ & $6(40,0)$ & $20(55,6)$ \\
\hline Aumento de peso & $9(42,9)$ & $4(26,7)$ & $13(36,1)$ \\
\hline Reducción de la libido & $9(42,9)$ & $5(33,3)$ & $14(38,9)$ \\
\hline Pesadillas & $6(28,6)$ & $3(20,0)$ & $9(25,0)$ \\
\hline Disfunción eréctil & $6(28,6)$ & $2(13,3)$ & $8(22,2)$ \\
\hline Hipotensión ortostática & $5(23,8)$ & & $5(13,9)$ \\
\hline Mareo & $5(23,8)$ & $1(6,7)$ & $6(16,7)$ \\
\hline Fatiga & $4(19,0)$ & $5(33,3)$ & $9(25,0)$ \\
\hline Hipercolesterolemia & $4(19,0)$ & $4(26,7)$ & $8(22,2)$ \\
\hline Cefalea & $3(14,3)$ & $2(13,3)$ & $5(13,9)$ \\
\hline Estreñimiento & $2(9,5)$ & $1(6,7)$ & $3(8,3)$ \\
\hline Dispepsia & $2(9,5)$ & & $2(5,6)$ \\
\hline Taquicardia & $2(9,5)$ & & $2(5,6)$ \\
\hline Somnolencia & $2(9,5)$ & $4(26,7)$ & $6(16,7)$ \\
\hline Rinitis & $1(4,8)$ & $1(6,7)$ & $2(5,6)$ \\
\hline Aumento de transaminasas & $1(4,8)$ & & $1(2,8)$ \\
\hline Hipertrigliceridemia & $1(4,8)$ & $3(20,0)$ & $4(11,1)$ \\
\hline Amenorrea & $1(4,8)$ & $1(6,7)$ & $2(5,6)$ \\
\hline Ginecomastia & $1(4,8)$ & & $1(2,8)$ \\
\hline Total RAM & 78 & 42 & 120 \\
\hline
\end{tabular}

A pesar de las RAM detectadas, la percepción de los pacientes es globalmente positiva ya que perciben una mejoría subjetiva de su enfermedad con el tratamiento. Un 95\% de los pacientes tratados con venlafaxina ha notado mejoría y efectividad en su tratamiento. En el caso de duloxetina ha sido un $81 \%$ los pacientes que han notado mejoría (14).

El $81 \%$ de los pacientes que son tratados con los antidepresivos estudiados presenta algún tipo de EA, lo que puede contribuir al incumplimiento del tratamiento. Esto hace necesario interrogar sobre estas molestias para valorar en qué medida influyen en la calidad de vida del paciente y modificar su actitud frente a la medicación (19).

\section{Antipsicóticos}

Sequedad de boca $(66,7 \%)$, aumento de peso $(42,9 \%)$ y reducción de que hacen hincapié en la importancia del control de la diabetes y en la aparición de ésta en pacientes tratados con olanzapina (21).

Resulta satisfactorio reflejar la efectividad percibida de estos tratamientos a pesar de los efectos adversos detectados. El 81\% de los pacientes que tienen prescrito olanzapina ha notado mejoría con el tratamiento, y es mejor aún en el caso de la quetiapina, con la que el $87 \%$ de los pacientes refiere la efectividad con el tratamiento. Por el contrario, otros artículos demuestran que el 64\% abandona el tratamiento por falta de eficacia (22).

\section{Limitaciones del estudio}

No pueden extraerse conclusiones categóricas debido al pequeño tamaño muestral de nuestro estudio. Sería interesante que otras farmacias participasen en estudios similares y tener una muestra mucho mayor para obtener datos relevantes.

Otra limitación es la falta de claridad en los resultados, ya que son pacientes tratados con gran cantidad de fármacos relacionados con el sistema nervioso central, los cuales producen EA muy similares. De hecho, un EA destacado en el caso de venlafaxina es la ansiedad y el exceso de sudoración, éstos son síntomas típicos de una persona con ansiedad que necesitaría dichos fármacos para encontrar mejoria, por lo que se puede confundir EA con falta de efectividad.

la libido $(42,9 \%)$ son las sospechas de RAM más frecuentes en olanzapina, seguidas de pesadillas y disfunción eréctil. En quetiapina son sequedad de boca $(40,0 \%)$, fatiga $(33,3 \%)$ y reducción de la libido (33,3\%), seguidas de somnolencia y aumento de peso. Con quetiapina el aumento de peso es menor y aparece en menor proporción de pacientes que a los que se les prescribe olanzapina (20).

El porcentaje de efectos adversos es menor en quetiapina $(12,21)$. También es importante señalar que la mayoría, como en el caso de los antidepresivos, son pacientes con polimedicación que amplifica la incidencia de estos efectos adversos.

No se han recogido datos de posible hiperglucemia en relación con olanzapina. Sí se ha detectado y reflejado un aumento de peso en el $42,9 \%$ de los pacientes, lo que se estima tiene relación con el aumento de glucosa en sangre. Hay referencias

\section{Aplicabilidad y utilidad}

de los resultados

El estudio ha permitido detectar sospechas de RAM en los fármacos ya nombrados y notificarlas a través de la red de farmacovigilancia, con lo que se consigue un incremento en la seguridad de su utilización seguridad.

El estudio también ha permitido informar a los pacientes sobre el uso adecuado de su medicación y acerca de los posibles efectos adversos, pudiendo solucionar alguno de ellos, favoreciendo así también la adhesión al tratamiento.

Los ensayos clínicos informan bien sobre la eficacia de los fármacos, pero poco acerca de la seguridad, por este motivo son necesarios estudios de recopilación de problemas de seguridad asociados al uso de medicamentos en la práctica clínica asistencial del farmacéutico comunitario. 


\section{Agradecimientos}

A todos los que me han ayudado en la realización de este trabajo, especialmente al Dr. Nicanor Floro Andrés Rodríguez, por dedicarme todo su tiempo y paciencia. A mi tutora, Dra. Pilar Modamio, por sus consejos, correcciones y ánimos. A la farmacéutica María del Pilar Rioboo Caridad, por mostrarse de acuerdo con el proyecto que he realizado en su farmacia. A mi familia, por apoyarme en cualquier situación.

\section{Referencias bibliográficas}

1. Cortes Españolas. Real Decreto $577 / 2013$, de 26 de Julio, por el que se regula la farmacovigilancia de medicamentos de uso humano. Boletín Oficial del Estado, $\mathrm{n}^{\circ} 179$, de 27/7/2013.

2. Madurga Sanz, M. Orígenes y fundamentos de la Atención Farmacéutica. Farmacovigilancia: evaluación de la seguridad. En: Mariño Hernández E. Máster en Atención Farmacéutica Integral. Barcelona: Universidad de Barcelona; 2013/2015. Págs. 111-147.

3. Cortes Españolas. Ley 44/2003, de 21 de noviembre, de ordenación de las profesiones sanitarias. Boletín Oficial del Estado, n 280, de 22/11/2003.

4. Instituto Nacional de Salud Mental [Internet]. Depresión. 2009. [Acceso 18/9/2015]. Disponible en: http:// www.nimh.nih.gov/health/publications/espanol/depresion/depresion_38791.pdf

5. Organización Panamericana de la Salud [Internet]. Tratamiento farmacológico de los trastornos mentales en la atención primaria de salud. Publicaciones de la OMS. 7-10. [Acceso 12/9/2015]. Disponible en: http//whqlibdoc.who. int/paho/2010/9789275331132_spa. pdF

6. Consejo General de Colegios Oficiales de Farmacéuticos. BOT Plus. Base de Datos del Conocimiento Sanitario. Madrid: CGCOF; 2015.
7. Agencia Española de Medicamentos y Productos Sanitarios [Internet]. Ficha técnica de Venlafaxina Combix. Revisado Abril 2013. [Acceso 12/9/2015]. Disponible en: http://www.aemps. gob.es/cima/pdfs/es/ft/66689/FT_ 66689.pdf

8. Agencia Española de Medicamentos y Productos Sanitarios [Internet]. Ficha técnica de Cymbalta. [Acceso 12/9/2015]. Disponible: http:// www.ema.europa.eu/docs/es_ES/ document_library/EPAR_-_Product_Information/human/000572/ WC500036781.pdf

9. Flórez J. Fármacos antidepresivos y antimaníacos. En: Flórez J (director), Armijo JA, Mediavilla A (directores asociados). Farmacología humana. $4^{\mathrm{a}}$ ed. Barcelona: Masson; 2003. Págs. 579-594.

10. Leucht S, Cipriani A, Spineli L, Mavridis D, Orey D, et al. Comparative efficacy and tolerability of 15 antipsychotic drugs in schizophrenia: a multiple-treatments meta-analysis. Lancet. 2013;382(9896):951-962. do:10.1016/S0140-6736(13)60733-3

11. Agencia Española de Medicamentos y Productos Sanitarios [Internet]. Ficha técnica de Olanzapina. (Zyprexa). [Acceso 12/9/2015]. http://www.ema. europa.eu/docs/es_ES/document_library/EPAR_-_Product_Information/ human/000287/WC500055611.pdf

12. Agencia Española de Medicamentos y Productos Sanitarios [Internet]. Ficha técnica de Quetiapina Actavis. [Acceso 14/9/2015]. Disponible en: http://www.aemps.gob.es/cima/pdfs/ es/ft/74231/FT_74231.pdf

13. Flórez J. Fármacos antipsicóticos neurolépticos. En: Flórez J (director), Armijo JA, Mediavilla A (directores asociados). Farmacología humana. $4^{\mathrm{a}}$ ed. Barcelona: Masson; 2003. p. 563577.

14. Heras Tabernero JA, Revuelta Bravo A. Hiperhidrosis localizada por fluoxetina. Actas Dermosifiliogr 2002;93(5):325-7. doi:10.1016/S00017310(02)76584-8
15. García-Parajuá P, Álvarez Iniesta I, Magariños M. Parkinsonismo reversible y dependiente de la dosis inducido por venlafaxina. Medicina Clínica 2003;120(19):756-759. doi:10.1016/ S0025-7753(03)75202-6

16. Baca Valdomero E, Cervera Enguix S, Grupo de estudio Teseo. Seguridad cardiovascular de venlafaxina retard en pacientes depresivos. Un estudio de farmacovigilancia. Psiquiatría Biológica. 2005;12(2):33-38.

17. Xue F, Strombom I, Turnbull B, Zhu S, Seeger J. Treatment with duloxetine in adults and the incidence of cardiovascular events. J Clin Psychopharmacol. 2012;32(1):23-30. doi:10.1097/ JCP. ob013e31823fb238

18. Arranz B. Duloxetina y trastorno de ansiedad generalizada, una buena asociación. Psiquiatría Biológica. 2014;21(1):38. doi:10.1016/j.psiq.2014.03.001

19. Soler Insa PA, Simón Martín O, Safont Lacal G. Efectos secundarios de los ISRS sin importancia para la salud que pueden contribuir al mal cumplimiento o al abandono del tratamiento. Psiquiatría Biológica. 2008;15 (4):101-108. doi:10.1016/S1134-5934(08)76480-7

20. Bernardo M, Vieta E, Saiz Ruiz J, Rico Villademoros F, Álamo C, Bobes J. Recomendaciones para el cambio de antipsicóticos. Posicionamiento de la Sociedad Espanola de Psiquiatría y Sociedad Española de Psiquiatría Biológica. Rev Psiquiatr Salud Ment (Barc.). 2011;4(3):150-168. doi:10.1016/j.rpsm.2011.07.003

21. Barceló Colomer ME, Mateu Ramon MC, Miralles Belda V, Moretó Reventós A. Olanzapina y descompensación diabética: a propósito de un caso. Aten Primaria. 2004; 34(1):58-59. doi:10.1016/S0212-6567(04)79455-X

22. San L, Arranz B, Perez V, Safont G, Corripio I, Ramirez N, et al. One-year, randomized, open trial comparing olanzapine, quetiapine, risperidone and ziprasidone effectiveness in antipsychotic-naive patients with a first-episode psychosis. Psychiatry Res. 2012;200:693-701. doi:10.1016/j.psychres.2012.07.005 